\title{
PMD \& DGD Performance Analysis in SMF due to Fiber Irregularities
}

\author{
A.M. Agarkar \\ Assistant Professor, \\ S.S.G.M.C.E. Shegaon (India)
}

\author{
Prajakta Joharapurkar \\ Student of M.E. (Digital Electronics) \\ S.S.G.M.C.E. Shegaon (India)
}

\begin{abstract}
A fundamental property of optical signal is polarization. Polarization refers to the electric field orientation of the light signal, which can vary significantly along the length of the fiber.

Signal energy at a given wavelength occupies two orthogonal polarization modes .A varying birefringence along its length will cause each polarization mode to travel at significantly different velocity and polarization orientation is related with distance.The resulting difference in propagation time, DGD between two orthogonal polarizations will result in pulse splitting. PMD is related to the differential group delay, DGD caused by birefringence in optical fibers.

Polarization mode dispersion, PMD is a phenomenon found in optical fiber and other wave guides .It is created by fiber irregularities and thus causes dispersion and distortion of the light pulse, thus increasing BER and limiting data rate.

There are various types of irregularities in fiber, which arise due to an asymmetric fiber core or can be introduced through internal stresses during fiber manufacture, or through external stresses during cabling and installation. Optical fiber manufacturing processes are designed to yield fibers with a circular cross-section. Any deviation from this form will generally result in an elliptical core, which in turn will result in a refractive index difference between the $\mathrm{X}$ and $\mathrm{Y}$-axes of the elliptical core. Even if the fiber core is manufactured with an ideal circular cross-section its refractive index can be asymmetric across its cross-section due to stresses built into the fiber during the manufacturing process or stress that is externally applied during deployment or operation.
\end{abstract}

In the present paper, authors have analyzed PMD and DGD performance in the single mode fiber with different fiber irregularities.

\section{General Terms}

Optical Fiber, Data Rates, SMF, Fiber Irregularities

\section{Keywords}

Optical fiber communication, Differential group delay (DGD), Polarization mode dispersion (PMD), BER, Data Rate.

\section{INTRODUCTION TO OPTICAL FIBER COMMUNICATION}

Fiber-optic communication is a method of transmitting information from one place to another by sending pulses of light through an optical fiber. The light forms an electromagnetic carrier wave that is modulated to carry information. First developed in the 1970s, fiber-optics communication systems have revolutionized the telecommunications industry and have played a major role in the advent of the Information Age. Because of its advantages over electrical transmission, optical fibers have largely replaced copper wire communications in core networks in the developed world.

The process of communicating using fiber-optics involves the following basic steps: Creating the optical signal involving the use a transmitter, relaying the signal along the fiber, ensuring that the signal does not become too distorted or weak, receiving the optical signal, and converting it into an electrical signal.

\subsection{System Components}

Modern fiber-optic communication systems generally include an optical transmitter to convert an electrical signal into an optical signal to send into the optical fiber, a cable containing bundles of multiple optical fibers that is routed through underground conduits and buildings, multiple kinds of amplifiers, and an optical receiver to recover the signal as an electrical signal. The information transmitted is typically digital information generated by computers, telephone systems, and cable television companies.

\subsection{Transmitters}

The role of an optical transmitter is to convert the electrical signal into optical form and to launch the resulting optical signal into the optical fiber. Figure shows the block diagram of an optical transmitter. It consists of an optical source, a modulator, and a channel coupler. Semiconductor lasers or light-emitting diodes are used as optical sources because of their compatibility with the optical-fiber communication channel. The optical carrier wave is modulated to generate the optical signal. Although an external modulator is sometimes used, it can be dispensed with in some cases, since the output of a semiconductor optical source can be modulated directly by varying the injection current. The coupler is typically a micro lens that focuses the optical signal onto the entrance plane of an optical fiber with the maximum possible efficiency.

The launched power is an important design parameter. One can increase the amplifier (or repeater) spacing by increasing it, but the onset of various nonlinear effects limits how much the input power can be increased. The launched power is often expressed in "dBm" units with $1 \mathrm{~mW}$ as the reference leve. The most commonly used optical transmitters are semiconductor devices such as light-emitting diodes (LEDs) and laser diodes. The difference between LEDs and laser diodes is that LEDs produce incoherent light, while laser diodes produce coherent light. Semiconductor optical transmitters are designed to be compact, efficient, and reliable, while operating in an optimal wavelength range, and directly modulated at high frequencies. 


\subsection{Receivers}

The main component of an optical receiver is a photo detector, which converts light into electricity using the photoelectric effect. The photo detector is typically a semiconductor-based photodiode. Several types of photodiodes include p-n photodiodes, $\mathrm{p}-\mathrm{i}-\mathrm{n}$ photodiodes, and avalanche photodiodes. Metal-semiconductor-metal (MSM) photo detectors are also used due to their suitability for circuit integration in regenerators and wavelength-division multiplexes.

The optical-electrical converters are typically coupled with a trans-impedance amplifier and a limiting amplifier to produce a digital signal in the electrical domain from the incoming optical signal, which may be attenuated and distorted while passing through the channel. Further signal processing such as clock recovery from data (CDR) performed by a phase-locked loop may also be applied before the data is passed on.

\subsection{Fiber}

An optical fiber is a flexible filament of very clear glass and is capable of carrying information in the form of light. This filament of glass is a little thicker than a human hair.

An optical fiber consists of a core, cladding, and a buffer (a protective outer coating), in which the cladding guides the light along the core by using the method of total internal reflection. The core and the cladding (which has a lower-refractive-index) are usually made of high-quality silica glass, although they can both be made of plastic as well. Connecting two optical fibers is done by fusion splicing or mechanical splicing and requires special skills and interconnection technology due to the microscopic precision required to align the fiber cores. [2].

\section{POLARIZATION, PMD \&DGD}

A fundamental property of optical signal is polarization. Polarization refers to the electric field orientation of the light signal, which can vary significantly along the length of the fiber.

Signal energy at a given wavelength occupies two orthogonal polarization modes. A varying birefringence along its length will cause each polarization mode to travel at significantly different velocity and polarization orientation is related with distance. The resulting difference in propagation time and DGD between two orthogonal polarizations will give rise to pulse splitting. PMD is related to the differential group delay whereas DGD caused by birefringence in optical fibers.

Polarization mode dispersion, PMD is a phenomenon found in optical fiber and other wave guides. It is created by fiber irregularities and thus causes dispersion and distortion of the light pulse, thus increasing BER thereby putting the upper limit on the data rate to be sent through the fiber.

Telecommunication network based on optical fiber technology have become a major information-transmission system, with high capacity optical fiber links encircling the globe in both terrestrial and undersea applications.

Because of its advantages over electrical transmission, the use of optical fiber has largely replaced copper wire communications in core networks in the developed world. Propagation of light through optical fiber is dependent on the nature of light and the structure of the optical fiber. Many optical fiber properties increase signal loss and reduce system bandwidth. Also because of different fiber structure and other external factor like temperature, stress etc. the effect such as Birefringence is responsible for Differential group delay (DGD) and PMD. [1]

\section{FIBER LOSSES AND PMD}

Attenuation is mainly a result of light absorption, scattering and bending losses. The intrinsic absorption loss is due to the material used to manufacture the fiber and hence puts the fundamental limit on the attenuation. Extrinsic absorption is due to different doping material used to vary the refractive index profile. Scattering losses are the results of the manufacturing processes and can be minimized using PCVD method. Bending losses are due to improper lying down the fiber cable through ducts and can be minimized by following the standard procedures.

One of the most important parameter that affects the system performance is the dispersion. Dispersion spreads the optical pulse as it travels along the fiber. This spreading of the signal pulse reduces the system bandwidth or the information-carrying capacity of the fiber. Dispersion limits how fast information is transferred. An error occurs when the receiver is unable to distinguish between input pulses caused by the spreading of each pulse.

In connection with the dispersion, polarization mode dispersion (PMD) is the most important parameter that affects the information charring capacity of the fiber. Keeping in view the present scenario of high demand for Bandwidth for internet and intranet applications, it is very necessary now-a-days to limit the system PMD of fiber. This ensures high bandwidth with the same fiber.

\section{BASICS OF FIBER PMD}

Modulated optical carrier signal propagates in an optical fiber in the form a pulsed modulated beam or wave of light. Light is a form of electro-magnetic radiation which is characterized by having a particular wavelength and frequency. The wavelength is defined as the distance between the points in the electromagnetic wave where the electric field has the highest amplitude. The frequency is defined as the number of complete wavelengths (or cycles) that traverse past a particular point in one second. The measure of frequency is Hertz $(\mathrm{Hz})$, or cycles per second. The electromagnetic spectrum consists of many different spectral regions defined by their respective wavelengths; for example the visible light that we are most accustomed to, ultra-violet light (UV) and the infra-red (IR) light that is actually used to transmit signals in fiber optic communications.

\section{TRANSMISSION BANDS}

The third transmission window centered on the wavelength 1.55 microns $(\mu \mathrm{m})$ or 1550 nanometers $(\mathrm{nm})$ is preferred for long distance communication using optical fibers because optical fiber exhibits the lowest attenuation at this wavelength, as shown in Fig.1. All electromagnetic waves are characterized by polarization, the direction in which the electric field (E) of the wave is oscillating. For example in Fig.2, the electric field of the light wave oscillates in the direction of $\mathrm{X}$-axis hence calling it as $\mathrm{X}$-polarized wave. Clearly, electromagnetic waves could have two polarizations along the $\mathrm{X}$ axis or along the $\mathrm{Y}$ axis, and if the 
electric field $\mathrm{E}$ is not aligned with either axis, the electromagnetic wave would contain both polarizations.

The speed at which a light wave travels through an optical medium (denoted as v) is dependent on the refractive index of the medium typically denoted as $\mathrm{n}$. The higher the refractive index the slower the speed of light and vice versa i.e. $v=c / n$ where $\mathrm{c}$ is a speed of light in vacuum.

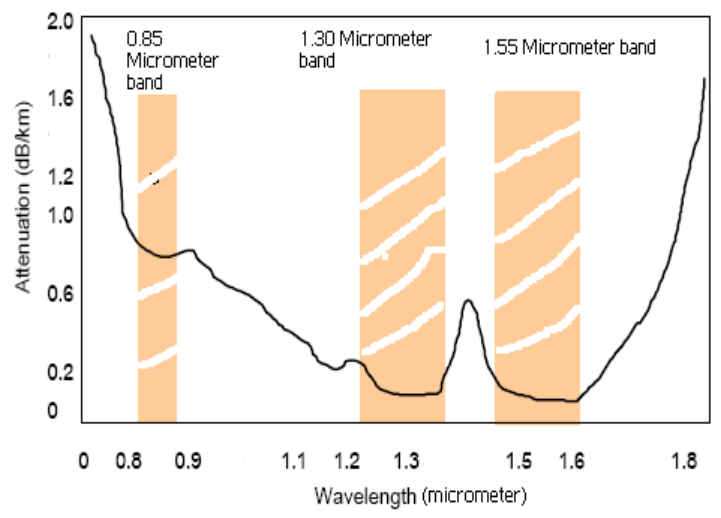

Fig. 1 Transmission Bands

\section{BIREFRINGENCE IN OPTICAL FIBER}

The refractive index ellipse is an ellipse whose axes are proportional to the refractive indices $n_{X}$ and $n_{Y}$. Clearly, for the case of a fiber with an absolutely symmetric refractive index profile, the refractive index ellipse becomes a circle. When the refractive indices in the $\mathrm{X}$ and $\mathrm{Y}$ directions are different then obviously the refractive index circle will become an ellipse, as shown in fig. 1due the effect called as birefringence. This splitting of light wave into two unequally reflected / transmitted wave by optically anisotropy medium is called birefringence. [5]
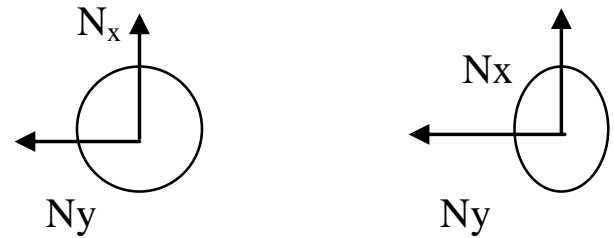

Fig.2 R.I.Variation and Polarization

In the figure 2, the refractive index $n_{X}$ is higher than $n_{Y}$, thus light polarized along the $\mathrm{X}$ axis propagates with a speed slower than that of the light polarized along the Y-axis. Typically the polarization that propagates with lower speed is called the "slow axis" whereas the polarization that propagates with the higher speed is called the "fast axis". The deviation of less than $1 \%$ in the circularity of the core can have noticeable effect on highspeed optical system. The birefringence of an optical fiber has the effect of slowing down the polarization state, which is oscillating along the slow axis with the higher refractive index (the $\mathrm{X}$-axis in Fig.3), relative to the polarization state oscillating along the Y-axis. Hence the birefringence in the fiber introduces a delay between two polarization states. This delay is known as the differential group delay (DGD) which is measured in picoseconds (ps) or 1 millionth of a millionth of a second $\left(10^{-12}\right.$ of a second). [1], [5].

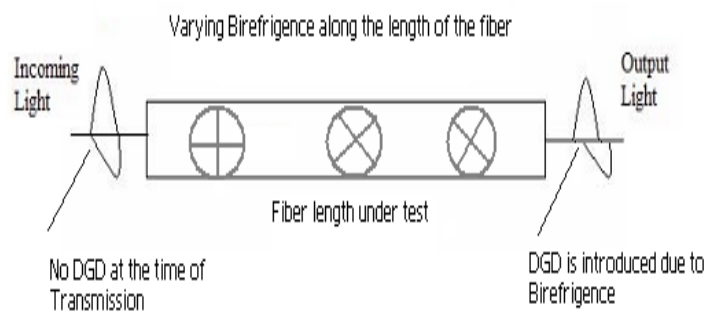

Fig. 3 Appearance of the DGD

The birefringence in the optical fiber slows down the $\mathrm{X}$ polarized state that sees the higher refractive index and causes a differential group delay (DGD) between the polarization states finally results in Pulse distortion often referred to as pulse splitting.

\section{POLARIZATION MODE DISPERSION}

A fundamental property of optical signal is its polarization. Polarization refers to the electric field orientation of the light signal, which can vary significantly along the length of the fiber. As shown in the figure 3, signal energy at a given wavelength occupies two orthogonal polarization modes. A varying birefringence along its length will cause each polarization mode to travel at a slightly difference velocity and polarization orientation is rotate with a distance .The resulting difference in propagation time $\Delta \mathrm{t}$ and DGD between two orthogonal polarization will result in pulse splitting. [2].

PMD is related to the differential group delay (DGD) caused by birefringence in optical fibers. It may be created by irregularities in the fiber, and causes dispersion and distortion of the light pulse as it propagates through the fiber. Research is being conducted to correct for this dispersion, which would allow for more information to be sent through the optical fiber at a higher speed.

The relationship between DGD and PMD is more complex due to the fact that fiber birefringence varies along its length, with different sections exhibiting different levels of birefringence both in terms of levels of refractive index asymmetry and the relative orientation of the slow and fast axis. Hence the fiber PMD cannot be approximated to the PMD of one section of the fiber. Thus to investigate the nature of PMD, it is necessary to represent the fiber as a series of concatenated birefringent sections of fiber separated by coupling sites, i.e. locations where the birefringence axes of one section are rotated with respect to the other .The birefringent fiber sections are orientated randomly relative to each other. At each coupling site the relative alignment of the axes of the birefringent sections can drift with respect to each other with time due to variations in the environmental conditions (e.g. temperature or external perturbation of the fiber). As a result the overall instantaneous value of DGD exhibited by the concatenated series of birefringent sections changes randomly with time. The average value of DGD is known as the PMD of the fiber. [3], [5]. 
The PMD of the fiber is proportional to the square root of the fiber length (L) multiplied by a proportionality coefficient. This coefficient is called the PMD coefficient (PMD coeffL) and is typically measured in units of picoseconds per square root kilometer ( $\mathrm{ps} / \mathrm{km} 1 / 2$ ). It is mathematically given as,

$$
\mathrm{PMD}=<\Delta \mathrm{t}>\text { PMDcoeff }^{\mathrm{L}}
$$

\section{THE IMPACT OF PMD \& DGD ON SYSTEM PERFORMANCE}

PMD causes a delay in the polarization components of the light. This causes the light pulse that enters the fiber to broaden, making it more difficult to distinguish the pulses once the light exits the fiber, as shown below in Fig.4 and Fig.5. It is clear that, the pulses are isolated for 2.5 Gbps system whereas it is almost mixed in $10 \mathrm{Gbps}$. The pulses begin to be broad enough that the peaks cross, making it impossible to decide from where each pulse begins and ends.

Hence, it is quite clear that polarization mode dispersion (PMD) is the major phenomenon found in optical fiber and other wave guides for reducing the bit rate. The reason behind it are the fiber irregularities and thus causes dispersion and distortion of the light pulse, thus increasing BER and limiting data rate.

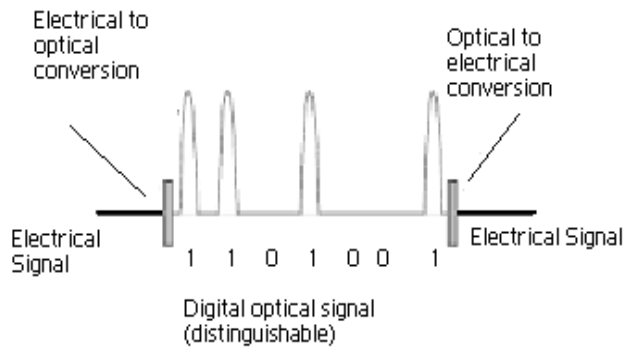

Fig. 4 FOC 2.5.Gbps System

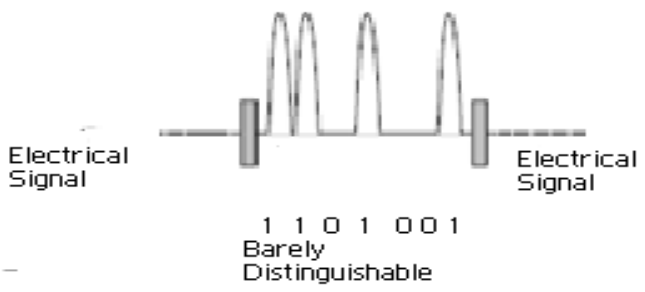

Fig.5 FOC 10 Gbps System

The impact of DGD will manifest at the receiver in the form of signal distortion. The distortion arises when the time slot (or bit period, $\mathrm{T}_{\mathrm{B}}$ ) of an individual pulse is stretched by the PMD induced delay to the point where the tail end of a leading pulse overlaps with the leading edge of a subsequent pulse. This is called Inter-Symbol Interference (ISI).

\section{SIMULATION RESULTS}

The results for irregularities such as stress or bending diameters of various values are given in the tables 1 and 2 given below.
Table1. Group Delay at various fiber irregularities (Bending Diameter : $60 \mathrm{~mm}$ )

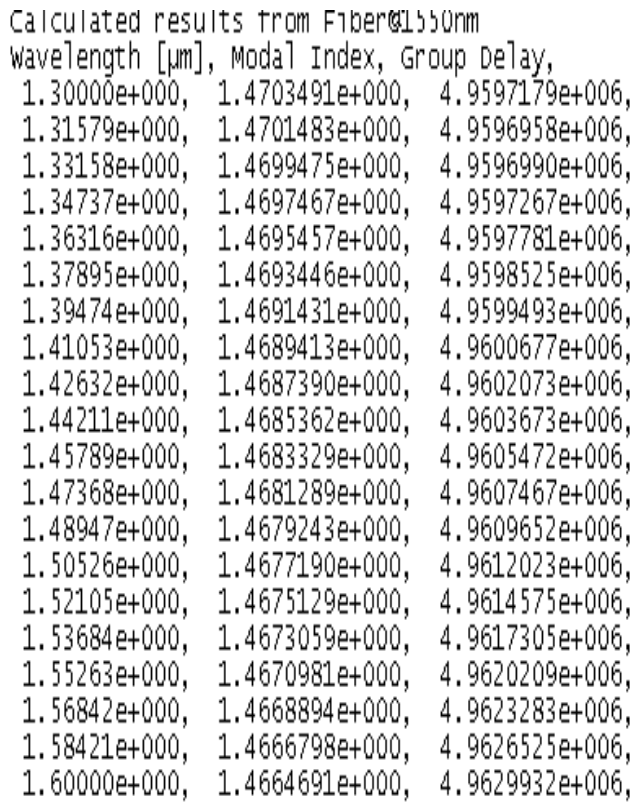

Table2. Waveguide dispersion, material dispersion and total dispersion at various fiber irregularities (Bending Diameter: $60 \mathrm{~mm}$ )

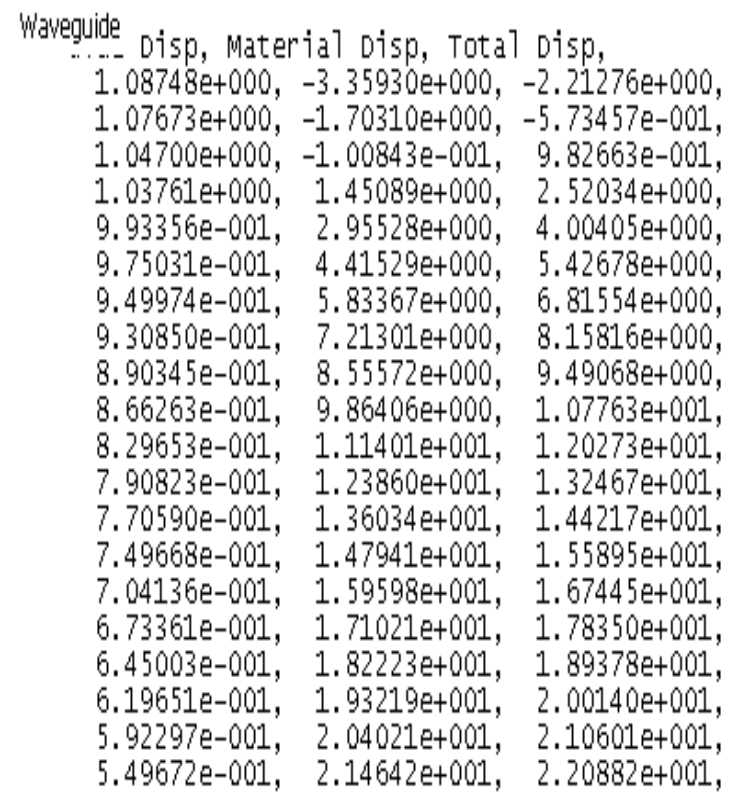

\subsection{Birefringence vs Wavelength Graph}

At,

a) R.I. of Core $(\eta 1)=1.45212$,

b) R.I. of Cladding $(\eta 2)=1.44692$,

c) Wavelength at $\lambda=1.30 \mu \mathrm{m}$

Fig. 6 shows that DGD versus wavelength graph. 


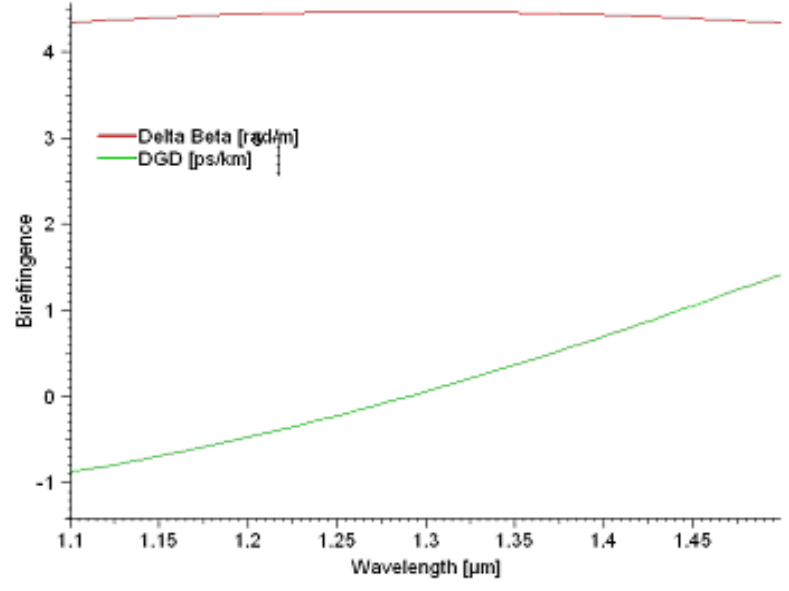

Fig. 6 DGD Vs Wavelength at $\lambda=1.30 \mu \mathrm{m}$

From this graph, it is found that, the DGD value for the central wavelength of $1.300 \mu \mathrm{m}$ is found to be $1.8347 \mathrm{ps} / \mathrm{km}$. Also as the wavelength increases further DGD values also increases for the above-mentioned parameters, $(\eta 1, \eta 2$ etc $)$.

\subsection{PMD vs Wavelength Graph}

PMD Performance of the fiber for the central wavelength of $1.300 \mu \mathrm{m}$ is shown in Fig. 7.

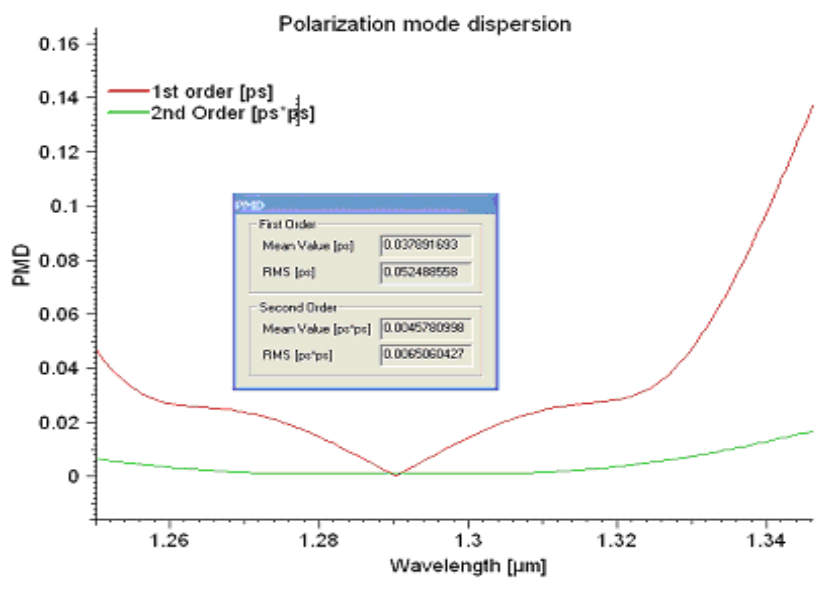

Fig 7: PMD Vs Wavelength at $\lambda=1.30 \mu \mathrm{m}$

At the central wavelength of $1.30 \mu \mathrm{m}$, the PMD values start decreasing initially from 0.04 to $0.01 \mathrm{ps}$ up to the wavelength of $1.29 \mu \mathrm{m}$. After that it increases. At the central wavelength of $1.3 \mu \mathrm{m}$ the value of PMD is about $0.03789 \mathrm{ps}$.

\subsection{Dispersion Vs wavelength Graph}

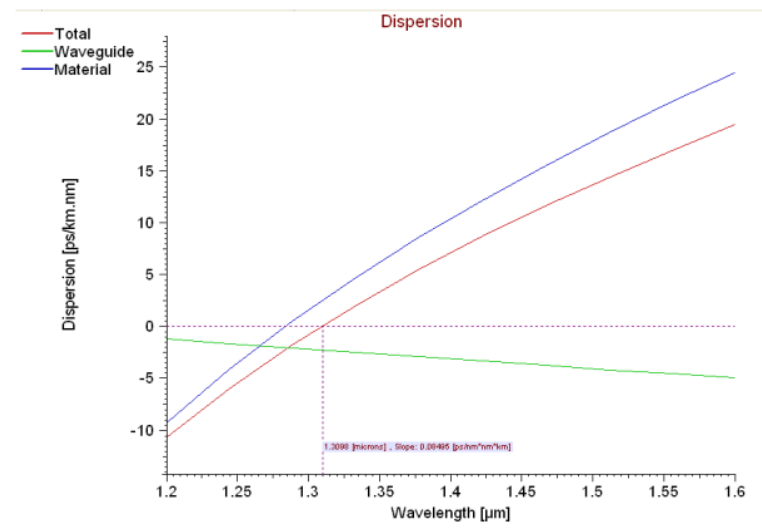

Fig 8 Wavelength vs Dispersion at $\lambda=1.30 \mu \mathrm{m}$

In fig. 8, the zero dispersion is obtained at 1.3098 micron with a slope of $0.08495 \mathrm{ps} / \mathrm{nm}^{*} \mathrm{~nm}$. The total Dispersion increases as the wavelength increases.

\subsection{DGD at $1.350 \mu m$}

From Fig. 9 it is found that, The DGD value for the central wavelength of $1.350 \mu \mathrm{m}$ is found to be $1.8297 \mathrm{ps} / \mathrm{km}$. Also as the wavelength increases further DGD values also increases for the above-mentioned parameters, $(\eta 1, \eta 2$ etc).

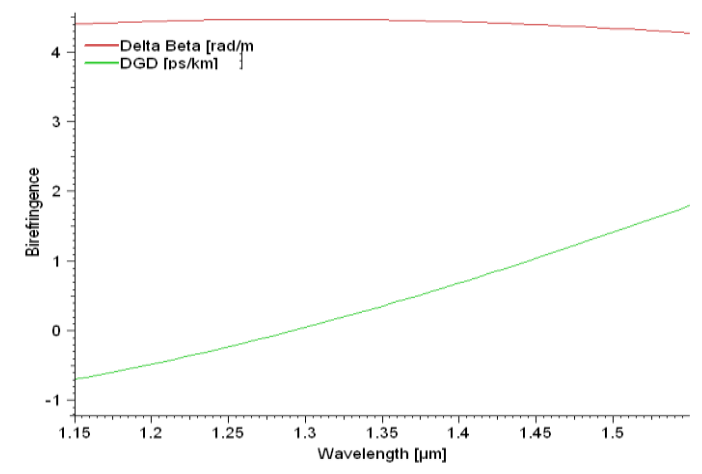

Fig 9 DGD Vs Wavelength at $\lambda=1.350 \mu \mathrm{m}$

\subsection{PMD vs wavelength graph}

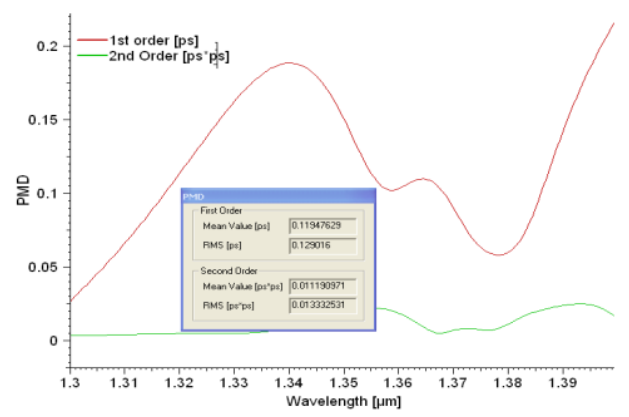

Fig.10: PMD Vs Wavelength at $\lambda=1.350 \mu \mathrm{m}$ 
From Fig.10, it is found that, for the central wavelength of $1.350 \mu \mathrm{m}$, the PMD value starts increaseing initially from 1.3 to $1.34 \mu \mathrm{m}$. After that it starts decreasing from $1.345 \mathrm{o} 1.36 \mu \mathrm{m}$. Actually PMD effects are random in nature, its value changes time to time depending upon external conditions also. Here at the central wavelength of $1.350 \mu \mathrm{m}$ the PMD value is found to be $0.6553 \mathrm{ps}$.

\subsection{Birefringence Vs Wavelength}

\section{Graph}

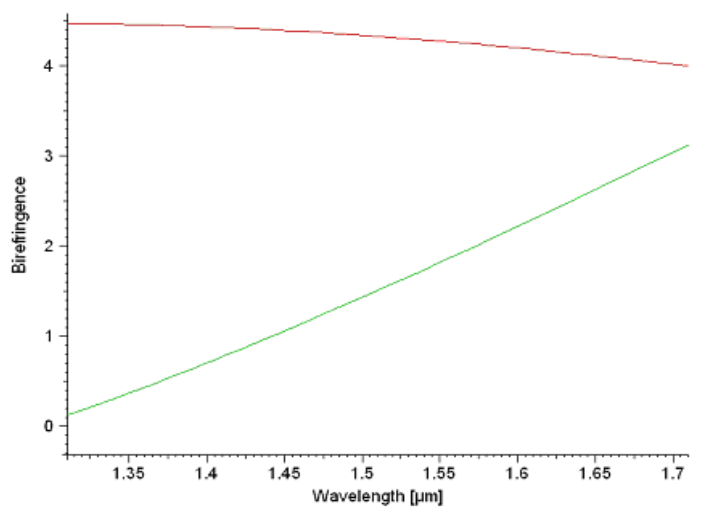

Fig 11: DGD Vs Wavelength at $\lambda=1.350 \mu \mathrm{m}$

From Fig.11, the DGD value for the central wavelength of $1.350 \mu \mathrm{m}$ is found to be $1.8297 \mathrm{ps} / \mathrm{km}$. Also as the wavelength increases further DGD values also increases for the above-mentioned parameters, ( $\eta 1, \eta 2$ etc).

\subsection{PMD vs Wavelength Graph}

Here it is observed that the value of PMD is random in nature at the wavelength of $1.510 \mu \mathrm{m}$ as compared to the PMD performance at 1.300 and $1.350 \mu \mathrm{m}$, In the second window PMD is random in nature but its value is quite less in comparison with it higher wavelength. Here for the central wavelength of 1.510 $\mu \mathrm{m}$ PMD value is 0.8144 ps.

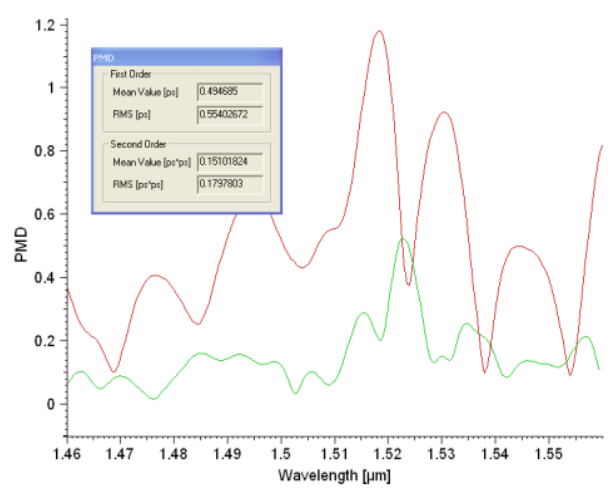

Fig 12: PMD Vs Wavelength at $\lambda=1.510 \mu \mathrm{m}$

\section{CONCLUSION}

About PMD in the fiber:

- PMD is related to DGD / Average Value of DGD.

- PMD is caused due to Birefringence.

- $\mathrm{PMD}$ is due to Asymmetries in fiber core.

- PMD causes Pulse Broadening.

- PMD is affected by Environment

- PMD is also known as Birefringence Dispersion.

- PMD can be reduced by using short fiber length, avoiding coiling of the fiber and by using DCF (dispersion compensated fiber).

The DGD and PMD Depends upon:

- Ellipticity of core

- Wavelength

- Bending radius

- Thermal Stress

- Temperature Variation

\section{REFERENCES}

[1] J. Sakai, and T. Kimura, "Birefringence Caused by Thermal Stress in Elliptically Deformed Core Optical Fibers", IEEE Journal of Quantum Electronics, vol. QE- 18, no. 11, pp 1899-1909, November 1982.

[2] Stein S.Vorbeck,E.Voges, "Polarization mode dispersion and polarization dependant loss in optical fiber system", University of Dortmund Germany www.photos.com.

[3] B.W.Hakki. Polarization mode dispersion in a single mode fiber. IEEE J.Lightwave technology Vol.14. No.10, Octomber 1996.

[4] I.P.Kaminow. Polarization in Optical fiber. IEEE Journal of .Quantom electronics, Vol QE-17, No.1, January 1981

[5] T.V.Muoi, " Receiver design for high-speed optical fiber systems," J.Lightwave Tch.,vol.LT-2, pp243267,June 1984.

[6] Geard Keiser.Optical fiber communication McGraw Hill Int. Editions.

[7] R.G.Swartz, "High performance integrated circuits for lightwave systems." in S.E.Miller and I.P.Kaminow, eds.,Optical Fiber Telecommunications II, Academic, New York, 1988. 